\title{
钼掺杂铜硅钙石的制备及其抗菌性能和细胞相容性研究
}

\author{
王恩典 ${ }^{1,2}$, 常 江 $^{1}$
}

(1. 中国科学院 上海硅酸盐研究所, 高性能陶瓷与超微结构国家重点实验室, 上海 200050; 2. 中国科学院大学 材料与光电研究中心，北京 100049)

摘 要: 含铜生物材料在高浓度铜离子释放时对细菌有优异的抑制作用, 但同时具有细胞毒性; 而在低浓度铜离子 释放时虽然具有良好的细胞相容性，但其抗菌性能低。因此，开发一种含铜生物材料，使其能够在高浓度铜离子释 放情况下同时具有优异抗菌性能和良好细胞相容性, 具有重要意义。本研究利用钼和铜之间的拮抗作用原理, 采用 溶胶一凝胶法制备了钼掺杂铜硅钙石，并通过细菌平板实验和细胞活力实验评价了材料的抗菌性能和细胞相容性。 实验结果显示, 钼掺杂铜硅钻石释放的高浓度铜离子(高于 $8.87 \mu \mathrm{g} \cdot \mathrm{mL}^{-1}$ )对金黄色葡萄球菌有良好的抑制性能。同 时, 由于铜钼离子的拮抗作用能降低高浓度铜离子对细胞的毒性, 钼掺杂铜硅钲石释放的铜离子在 $9.65 \mu \mathrm{g} \cdot \mathrm{mL}^{-1}$ 的 高浓度下依然能保证人脐静脉内皮细胞存活率高达 $90 \%$ 。因此，钼掺杂可以作为含铜生物材料降低其细胞毒性的 一种有效途径，为开发低细胞毒性含铜抗菌生物材料提供了新的路径。

关 键 词: 铜; 生物材料; 钼; 掺杂; 铜硅钙石; 抗菌性能; 细胞相容性

中图分类号: TQ174 文献标志码: A

\section{Mo Doped Cuprorivaite: Preparation, Antibacterial Performance and Cytocompatibility}

\author{
WANG Endian ${ }^{1,2}$, CHANG Jiang
}

(1. State Key Laboratory of High Performance Ceramics and Superfine Microstructure, Shanghai Institute of Ceramics, Chinese Academy of Sciences, Shanghai 200050, China; 2. Center of Materials Science and Optoelectronics Engineering, University of Chinese Academy of Sciences, Beijing 100049, China)

\begin{abstract}
Copper-containing biomaterials have excellent inhibitory effect on bacteria growth by releasing copper ions at high concentration, which may have cytotoxicity at the same time. Although low concentration of copper ions has good cytocompatibility, the antibacterial activity is unsatisfactory. Therefore, it is of great significance to develop copper- containing biomaterials, which have excellent antibacterial property and good cytocompatibility. In this study, on considering the antagonistic effect between copper and molybdenum, molybdenum doped cuprorivaite (Mo-Cup) was synthesized by Sol-Gel method and its antibacterial properties and cell compatibility were evaluated by bacterial plate experiment and cell activity assay. The results showed that copper ions with high concentration (above $8.87 \mu \mathrm{g} \cdot \mathrm{mL}^{-1}$ ) released from Mo-Cup had a good inhibitory effect on Staphylococcus aureus. In addition, because the antagonistic effect between copper and molybdenum ions released from Mo-Cup can reduce the cytotoxicity of high concentration of copper ions, the survival rate of umbilical vein endothelial cells (HUVECs) was as high as 90\% in the extracts of Mo-Cup at a high $\mathrm{Cu}$ ion concentration $\left(9.65 \mu \mathrm{g} \cdot \mathrm{mL}^{-1}\right)$. Therefore, molybdenum doping can be considered as an
\end{abstract}

收稿日期：2020-08-31；收到修改稿日期：2020-10-28; 网络出版日期：2020-11-05

基金项目: 国家重点研发计划(2016YFC1100201) National Key Research and Development Plan of China (2016YFC1100201)

作者简介：王恩典(1991-)，男，博士研究生. E-mail: wangendian@student.sic.ac.cn WANG Endian (1991-), male, PhD candidate. E-mail: wangendian@student.sic.ac.cn

通信作者：常 江，研究员. E-mail: jchang@mail.sic.ac.cn CHANG Jiang, professor. E-mail: jchang@mail.sic.ac.cn 
effective approach to reduce the cytotoxicity of copper-containing biomaterials for the development of low-toxic antibacterial biomaterials.

Key words: $\mathrm{Cu}$; biomaterial; Mo; doping; cuprorivaite; bacteria inhibition; cytocompatibility

在组织修复过程中抑制组织缺损处细菌的生长 可以加快修复过程 ${ }^{[1-2]}$ 。在众多抗菌生物材料中, 无 机抗菌生物材料因具有优异的稳定性、高效性、广 谱性而受到了广泛关注, 其中含铜生物材料既能有 效抑制大肠杆菌和金黄色葡萄球菌 ${ }^{[3-4]}$, 又能促进 血管内皮细胞血管化 ${ }^{[5-7]}$, 已经在抗细菌感染的组织 修复中得到广泛研究。然而抑制细菌生长的铜离子 浓度须高于 $6.4 \mu \mathrm{g} \cdot \mathrm{mL}^{-1[3]}$, 而保障血管内皮细胞良 好存活的铜离子浓度须在 $0.32 \sim 3.2 \mu \mathrm{g} \cdot \mathrm{mL}^{-1[7-8]}$ 之内, 高浓度下铜离子虽然具有抗菌活性, 但同时也具有 细胞毒性。因此, 开发一种含铜生物材料在释放相 同铜离子浓度下兼具优异的抗菌性能和良好的细胞 相容性具有重要意义。

微量元素 Mo 是动物必需的微量元素, Mo 进入机 体后, 主要参与两个方面的物质代谢过程: 一是作为 硝酸还原酶、黄嘌呤氧化酶和脱氢酶等酶的辅助因子 参与许多生化反应; 二是以无机盐的形式与其他营养 元素发生相互作用, 特别是与铜离子在生理环境中存 在拮抗作用 ${ }^{[9-12]}$ 。四硫代钼酸铵 $\left.\left(\mathrm{NH}_{4}\right)_{2} \mathrm{MoS}_{4}\right)$ 作为铜离 子螯合剂, 能够和铜离子以及细胞内的伴铜蛋白和 铜转运蛋白形成三元复合物阻止铜离子向细胞内转 运, 这已在医治威尔逊氏病、恶性肿瘤、铜积累导致 的肝脏和神经系统疾病中得到应用 ${ }^{[13-16]}$ 。

基于钼铜的拮抗作用，在含铜生物材料中掺杂 钼有可能使材料在保持抗菌性能的同时, 降低其对 细胞的毒性。本研究将钼离子引入含铜生物材料铜 硅钙石 $\left(\mathrm{CaCuSi}_{4} \mathrm{O}_{10}, \mathrm{Cup}\right)$ 中, 评价钼离子通过拮抗 效应对减弱高浓度铜离子细胞毒性的作用, 以及钼 掺杂铜硅钙石(Mo-Cup)在高浓度铜离子释放下的抗 菌性能和细胞相容性。

\section{1 实验方法}

\section{1 实验药品}

实验采用的三水合硝酸铜 $\left(\mathrm{Cu}\left(\mathrm{NO}_{3}\right)_{2} \cdot 3 \mathrm{H}_{2} \mathrm{O}\right)$ 、四 水合硝酸钙 $\left(\mathrm{Ca}\left(\mathrm{NO}_{3}\right)_{2} \cdot 4 \mathrm{H}_{2} \mathrm{O}\right)$ 、四水合七钼酸铵 $\left(\left(\mathrm{NH}_{4}\right)_{6} \mathrm{Mo}_{7} \mathrm{O}_{24} \cdot 4 \mathrm{H}_{2} \mathrm{O}\right)$ 、正硅酸乙酯 $\left(\left(\mathrm{C}_{2} \mathrm{H}_{5} \mathrm{O}\right)_{4} \mathrm{Si}(\mathrm{TEOS})\right)$ 、 浓硝酸 $\left(\mathrm{HNO}_{3}\right)$ 、五水合硫酸铜 $\left(\mathrm{CuSO}_{4} \cdot 5 \mathrm{H}_{2} \mathrm{O}\right)$ 均为分 析纯, 购自中国医药集团上海化学试剂有限公司。

\section{2 材料的合成}

实验采用溶胶一凝胶法制备钼掺杂铜硅钙石。将
摩尔比例为 $1: 0.16: 8$ 的 $\left(\mathrm{C}_{2} \mathrm{H}_{5} \mathrm{O}\right)_{4} \mathrm{Si}(\mathrm{TEOS}) 、 \mathrm{HNO}_{3}$ $\left(2 \mathrm{~mol} \cdot \mathrm{mL}^{-1}\right)$ 、去离子水搅拌 $0.5 \mathrm{~h}$ 制备硅溶胶。将 不同含量 $(5 \%$ 、 $10 \%)$ 的钼源 $\left(\left(\mathrm{NH}_{4}\right)_{6} \mathrm{Mo}_{7} \mathrm{O}_{24} \cdot 4 \mathrm{H}_{2} \mathrm{O}\right)$ 、 铜源 $\left(\mathrm{Cu}\left(\mathrm{NO}_{3}\right)_{2} \cdot 3 \mathrm{H}_{2} \mathrm{O}\right)$ 、钙源 $\left(\mathrm{Ca}\left(\mathrm{NO}_{3}\right)_{2}\right)$ 、硅溶胶混 合于烧杯中并搅拌 $5 \mathrm{~h}$, 密封后在 $60{ }^{\circ} \mathrm{C}$ 陈化 $24 \mathrm{~h}$, $120{ }^{\circ} \mathrm{C}$ 干燥 $48 \mathrm{~h}$ 。随后将所得样品在 $1000{ }^{\circ} \mathrm{C}$ 炦烧 $3 \mathrm{~h}$ 后, 采用行星球磨机球磨 $4 \mathrm{~h}$ 过 200 目 $(80 \mu \mathrm{m})$ 篮。

\section{3 材料的性能表征}

采用 $18 \mathrm{~kW}$ 转靶 X 射线衍射仪(XRD)分析钼掺 杂铜硅钙石粉体的物相组成; 使用场发射扫描电子 显微镜(SU8200)观察粉体的微观形貌及元素分布; 利用电感耦合等离子体发射光谱仪(ICP-OES)检测 粉体在细菌培养基、细胞培养液中 $\mathrm{Cu} 、 \mathrm{Mo} 、 \mathrm{Si}$ 离 子的释放量。

\section{4 抗菌实验}

实验采用菌落计数法研究铜离子、铜钼离子组合 及钼掺杂铜硅钙石粉体细菌培养基浸提液对金黄色葡 萄球菌(Staphylococcus aureus, S.A.)的增殖抑制效果。

a)离子抗菌操作步骤: 用细菌培养基将五水合 硫酸铜 $\left(\mathrm{CuSO}_{4} \cdot 5 \mathrm{H}_{2} \mathrm{O}\right)$ 、四水合七钼酸铵 $\left(\left(\mathrm{NH}_{4}\right)_{6} \mathrm{Mo}_{7} \mathrm{O}_{24} \cdot 4 \mathrm{H}_{2} \mathrm{O}\right)$ 配制成浓度为 $10 \mu \mathrm{g} \cdot \mathrm{mL}^{-1}$ 的铜 离子、钼离子及铜钼离子组合溶液; 取 $1 \mathrm{~mL}$ 离子溶 液、 $1 \mathrm{~mL}$ 细菌培养基(作为空白对照)加入 $0.1 \mathrm{~mL}$ OD (Optical density, $600 \mathrm{~nm}$ ) 值为 0.01 的 S.A.细菌悬 液共培养 $12 \mathrm{~h}$; 将共培养后菌液用 PBS 缓冲溶液稀 释 $10^{4}$ 倍后取 $25 \mu \mathrm{L}$ 均匀涂于琼脂培养板上培养 $16 \mathrm{~h}$, 拍照后统计菌落个数。每组三个平行样。

b)浸提液抗菌步骤: 以 $200 \mathrm{mg} \cdot \mathrm{mL}^{-1}$ 的比例将 钼掺杂铜硅钻石粉体加入到细菌培养基中, 密封后 置于 $37{ }^{\circ} \mathrm{C}$ 恒温摇床浸提 $24 \mathrm{~h}$, 静置后取上清液用 $0.22 \mu \mathrm{m}$ 滤膜过滤后得到 $200 \mathrm{mg} \cdot \mathrm{mL}^{-1}$ 的钿掺杂铜硅 钙石细菌培养基标准浸提液。再将其按需要用细菌 培养基进行梯度稀释。取每种粉体细菌培养基浸提 液溶液 $1 \mathrm{~mL}$ 、细菌培养基 $1 \mathrm{~mL}$ (作为空白对照), 加 入 $0.1 \mathrm{~mL} \mathrm{OD}$ 值为 0.01 的 S.A. 细菌悬液共培养 $12 \mathrm{~h}$; 将共培养后菌液用 PBS 缓冲溶液稀释 $10^{4}$ 倍后取 $25 \mu \mathrm{L}$ 均匀涂于琼脂培养板上培养 $16 \mathrm{~h}$, 后续操作 步骤同步骤 a)。

\section{5 细胞实验}

实验采用人真皮成纤维细胞 (Human Dermal 
Fibroblasts, HDFS)和人脐静脉内皮细胞(Human Umbilical Vein Endothelial Cells, HUVECs)研究铜离子、 铜钼离子组合及钼掺杂铜硅钙石粉体细胞浸提液的 细胞相容性。

a)含铜离子及铜钼离子组合的细胞培养液由五 水合硫酸铜 $\left(\mathrm{CuSO}_{4} \cdot 5 \mathrm{H}_{2} \mathrm{O}\right)$ 、四水合七钼酸铵 $\left(\left(\mathrm{NH}_{4}\right)_{6} \mathrm{Mo}_{7} \mathrm{O}_{24} \cdot 4 \mathrm{H}_{2} \mathrm{O}\right)$ 和细胞培养液配制并稀释至 所需浓度。

b)钼掺杂铜硅钙石细胞培养液浸提液制备过程 如下: 以 $200 \mathrm{mg} \cdot \mathrm{mL}^{-1}$ 的比例将钼掺杂铜硅钙石粉 体加入到相应细胞培养液中, 密封后置于 $37{ }^{\circ} \mathrm{C}$ 恒 温摇床浸提 $24 \mathrm{~h}$, 静置后取上清液用 $0.22 \mu \mathrm{m}$ 滤膜 过滤后得到 $200 \mathrm{mg} \cdot \mathrm{mL}^{-1}$ 的钼掺杂铜硅钙石细胞 培养液标准浸提液, 随后将标准浸提液按需要进行 梯度稀释。

用不同浓度的离子溶液和钼掺杂铜硅钙石细胞 浸提液将两种细胞在 $37{ }^{\circ} \mathrm{C}$, 二氧化碳 $\left(\mathrm{CO}_{2}\right)$ 浓度为 $5 \%$ 的细胞培养箱中培养, 隔天更换细胞培养液。在 预设时间点采用 Cell Counting Kit-8 试剂盒对细胞 活性进行检测。

所有细菌和细胞实验的各组数据均经 $t$ 检验后, $p<0.05$ 和 $p<0.01$ 时, 将两组数据分别看作有显著差 异和极显著差异。

\section{2 结果与讨论}

\section{1 钼掺杂铜硅钙石的性能表征}

本研究系统评价了钼掺杂铜硅钲石(Mo-Cup)的
物相组成、表面形貌、元素分布、离子释放。通过 XRD 对钼掺杂铜硅钙石的物相进行了表征。Mo-Cup 大部分衍射峰均和标准卡片 PDF\#85-0158 $\left(\mathrm{CaCuSi}_{4} \mathrm{O}_{10}\right.$, Cup)保持一致(图 1(a)), 5\%钼掺杂铜硅钙石(5Mo-Cup) 和 $10 \%$ 钼掺杂铜硅钻石 (10Mo-Cup)均出现了 $\mathrm{SiO}_{2}$ 物相, 并且随着钼元素掺入量的增加, 10Mo-Cup 中 出现了 $\mathrm{CaMoO}_{4}$ 物相。使用扫描电镜对粉体的表面 形貌进行了观察, 图 1(b,c)分别为 5Mo-Cup 和 10Mo-Cup 粉体的微观形貌。粉体有轻微的团聚, 粒 径分布在几百纳米到几微米之间。对粉体进行元素 面扫描分析发现(图 2), 钼掺杂铜硅钻石中的确存 在钼元素, 且各元素均匀分布。

为了评价钼掺杂铜硅钙石的离子释放行为, 本 研究对不同钼掺杂量铜硅钲石和铜硅钻石在细菌培 养基(BCM)和细胞培养液(ECM)中铜离子、钼离子、 硅离子的释放量进行了检测。硅离子和钼离子在细菌 培养基和细胞培养液中释放量接近(图 1(d f)), 而 铜离子在细菌培养基浸提液释放量都高于细胞培养 液浸提液的释放量, 这是由于铜离子与细胞培养液 缓冲体系的磷酸根存在溶解平衡, 不利于铜离子释 放。综合来看, 10Mo-Cup 和 Cup 的铜离子和硅离子 释放量接近, 为了方便研究钼掺杂后对铜硅钙石抗菌 性能和细胞相容性的影响, 本研究选取 10Mo-Cup 进 行抗菌试验和细胞活力实验。

\section{2 铜离子及铜钼离子组合的抗菌性能和细} 胞相容性

图 3 为不同浓度铜离子 $\left(\mathrm{CuSO}_{4} \cdot 5 \mathrm{H}_{2} \mathrm{O}\right.$ 溶液 $)$ 和钼 离子溶液 $\left(\left(\mathrm{NH}_{4}\right)_{6} \mathrm{Mo}_{7} \mathrm{O}_{24} \cdot 4 \mathrm{H}_{2} \mathrm{O}\right.$ 溶液 $)$ 对 $\mathrm{S} . \mathrm{A}$. 作用后
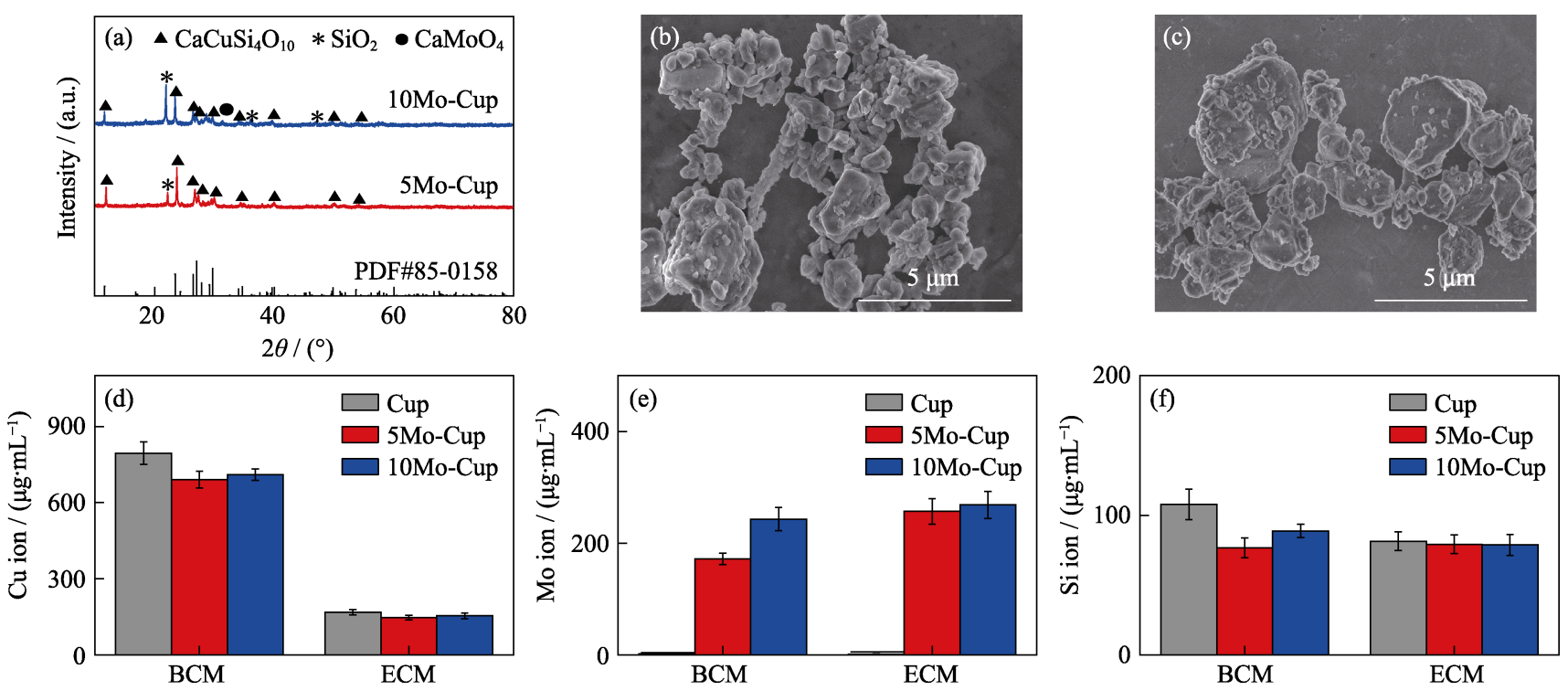

图 1 钼掺杂铜硅钻石的性能

Fig. 1 Properties of Mo doped cuprorivaite (Mo-Cup)

(a) XRD patterns, SEM images of (b) 5Mo-Cup and (c) 10Mo-Cup, and content of (d) copper ion, (e) molybdenum ion and (f) silicon ion released from Cup, 5Mo-Cup and 10Mo-Cup in bacterial culture medium (BCM) and cell culture medium (ECM) 

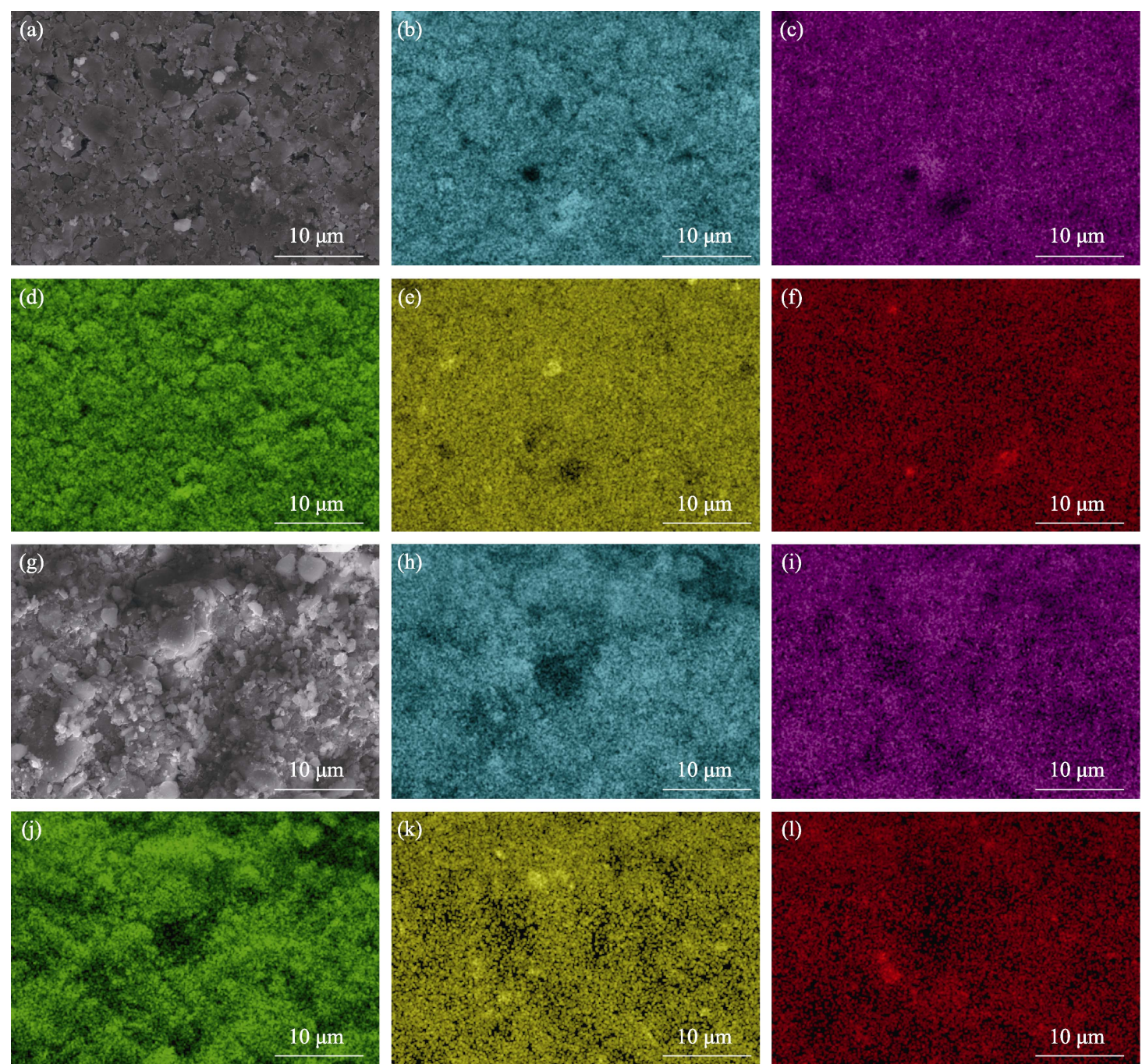

图 2 钼掺杂铜硅钻石( $a \sim f) 5 \mathrm{Mo}-\mathrm{Cup}$ 和( $\mathrm{g} \sim 1) 10 \mathrm{Mo}-\mathrm{Cup}$ 的(a,g)SEM 照片及其(b f, h l)元素分布图

Fig. 2 (a,g) SEM images of (a-f) 5Mo-Cup and (g-l) 10Mo-Cup and (b-f, h-l) corresponding elemental maps (b, h): $\mathrm{Si} ;(\mathrm{c}, \mathrm{i}): \mathrm{Ca} ;(\mathrm{d}, \mathrm{j}): \mathrm{O} ;(\mathrm{e}, \mathrm{k}): \mathrm{Cu} ;(\mathrm{f}, \mathrm{l}): \mathrm{Mo}$

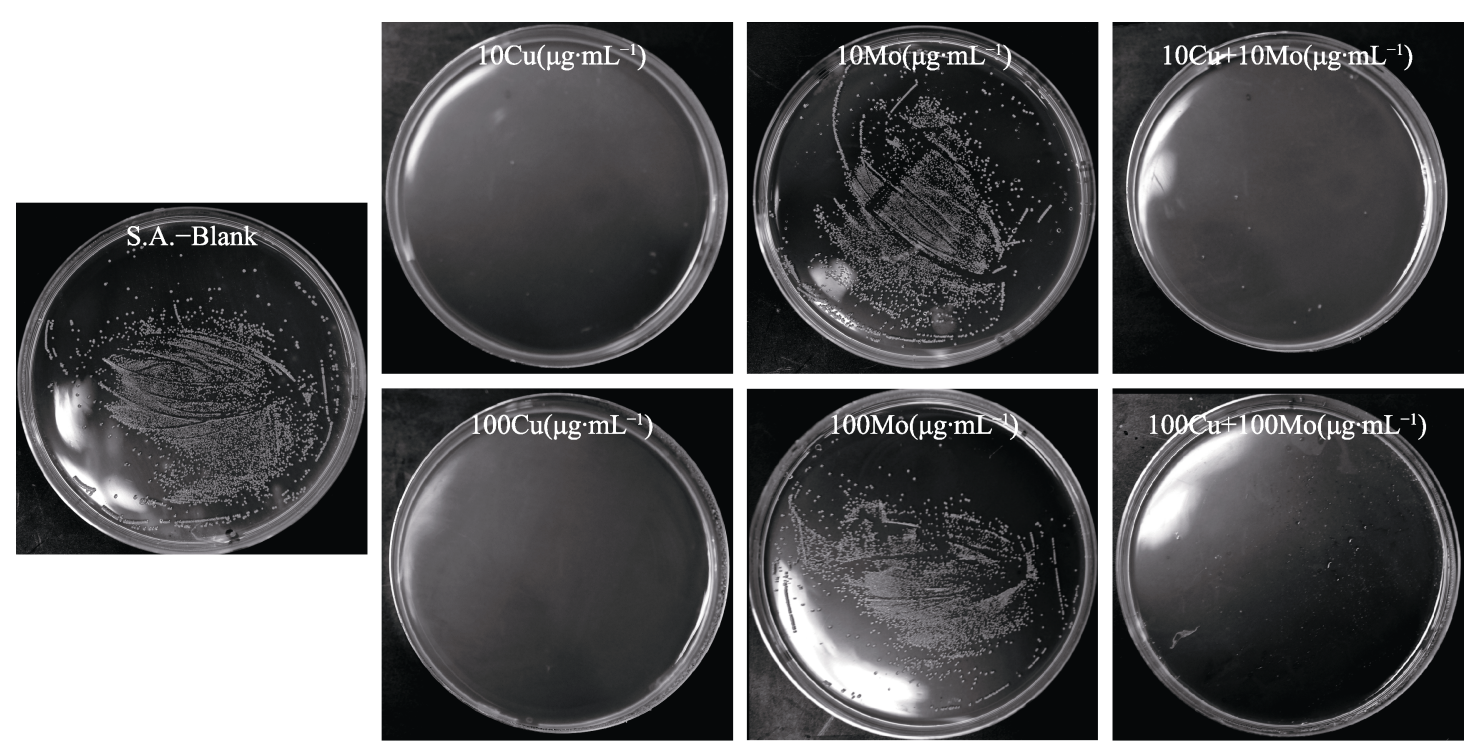

图 3 不同浓度铜离子溶液和钿离子溶液对金黄色葡萄球菌的作用

Fig. 3 Effect of different concentrations of copper ion $\left(\mathrm{CuSO}_{4} \cdot 5 \mathrm{H}_{2} \mathrm{O}\right)$ solution and molybdenum ion $\left(\left(\mathrm{NH}_{4}\right)_{6} \mathrm{Mo}_{7} \mathrm{O}_{24} \cdot 4 \mathrm{H}_{2} \mathrm{O}\right)$ solution on Staphylococcus aureus 
的细菌数量, 发现铜离子浓度为 10 和 $100 \mu \mathrm{g} \cdot \mathrm{mL}^{-1}$ 时铜离子溶液对 S.A. 的抑制率为 $100 \%$, 钼离子浓 度为 $100 \mu \mathrm{g} \cdot \mathrm{mL}^{-1}$ 时并未对金黄色葡萄球菌产生抑 制作用。在铜离子和钼离子都为 10 或 $100 \mu \mathrm{g} \cdot \mathrm{mL}^{-1}$ 时其组合溶液对金黄色葡萄球菌抗菌率依然为 $100 \%$, 说明钼离子不影响铜离子对金黄色葡萄球 菌的抑制。

为验证钼离子是否能够拮抗高浓度铜离子对细 胞的毒性, 本研究探讨了铜离子、铜钼离子组合对 人真皮成纤维细胞 (HDFs) 和人脐静脉内皮细胞 (HUVECs) 活力的影响。图 4(a) 为含钼离子 $\left(\mathrm{NH}_{4}\right)_{6} \mathrm{Mo}_{7} \mathrm{O}_{24} \cdot 4 \mathrm{H}_{2} \mathrm{O}$ 培养液对 HDFs 和 HUVECs 活 力的影响。HDFs 的存活结果显示, $2 \sim 10 \mu \mathrm{g} \cdot \mathrm{mL}^{-1}$ 浓 度的钼离子的细胞活力水平与空白对照(Blank)组 相当; HUVECs 的存活结果显示, 培养 1 和 $3 \mathrm{~d}$ 时, 随着钼离子浓度的增加, 钼离子对细胞活力有一定 的抑制; 培养 $5 \mathrm{~d}$ 时, 不同浓度钼离子的细胞活力水 平与 Blank 组水平相当。图 4(b)为含钼离子和铜离 子培养液(含 $\mathrm{CuSO}_{4} \cdot 5 \mathrm{H}_{2} \mathrm{O}$ 和 $\left(\mathrm{NH}_{4}\right)_{6} \mathrm{Mo}_{7} \mathrm{O}_{24} \cdot 4 \mathrm{H}_{2} \mathrm{O}$ 细
胞培养液)对 HDFs 和 HUVECs 活力的影响。HDFs 组的存活结果显示, 第 1 天, $10 \mu \mathrm{g} \cdot \mathrm{mL}^{-1}$ 的铜离子对 HDFs 产生一定抑制作用, 但仍能维持 55\% Blank 组 水平。加入不同浓度钼离子后, HDFs 活力水平与 Blank 组相当, 第 3 天和第 4 天, 各组与 Blank 组活 力水平相当。 HUVECs 的存活结果显示, $10 \mu \mathrm{g} \cdot \mathrm{mL}^{-1}$ 铜离子对 HUVECs 有明显毒性, 第 3 天和第 5 天, 该 组的 HUVECs 存活率低于 $40 \%$, 而在 $10 \mu \mathrm{g} \cdot \mathrm{mL}^{-1}$ 铜 离子培养液中加入不同浓度钼离子后, HUVECs 的 存活率都有了明显提升。因此, $10 \mu \mathrm{g} \cdot \mathrm{mL}^{-1}$ 铜离子对 HUVECs 有明显毒性, 引入钼离子, 即使在最低浓 度 $2 \mu \mathrm{g} \cdot \mathrm{mL}^{-1}$ 钼离子时也能够显著减弱铜离子对 HUVECs 的毒性。

Ning 等 ${ }^{[3]}$ 报道铜离子对大肠杆菌和金黄色葡萄 球菌的最小抑制浓度为 $6.4 \mu \mathrm{g} \cdot \mathrm{mL}^{-1}$, 同时 $\mathrm{Kong}^{[7-8]}$ 报道铜离子的浓度高于 $3.2 \mu \mathrm{g} \cdot \mathrm{mL}^{-1}$ 时, HUVECs 的 存活率会低于 $60 \%$ 。为了探究钼离子是否能够降低 在优异抗菌浓度下铜离子对 HUVECs 的毒性, 本研 究选择略高于最小抗菌浓度 $\left(10 \mu \mathrm{g} \cdot \mathrm{mL}^{-1}\right)$ 的铜离子


图 4 含 $10 \mu \mathrm{g} \cdot \mathrm{mL}^{-1}$ 铜离子培养液、含钼离子细胞培养液及铜钼组合培养液对细胞活力的影响

Fig. 4 Effects of culture medium containing $10 \mu \mathrm{g} \cdot \mathrm{mL}^{-1}$ copper ion, molybdenum ion and copper-molybdenum ions on cell activity assay

(a) Effects of molybdenum ions on the proliferation of human dermal fibroblast (HDFs) and human umbilical vein endothelial cells (HUVECs)( $x$ Mo stands for medium containing $x \mu \mathrm{g} \cdot \mathrm{mL}^{-1}$ molybdenum ion; $x=2,4,6,8,10$ ); (b) Effects of copper molybdenum ions combination on the proliferation of HDFs and HUVECs $\left(10 \mathrm{Cu}+y\right.$ Mo stands for medium containing $10 \mu \mathrm{g} \cdot \mathrm{mL}^{-1}$ copper ions and $y \mu \mathrm{g} \cdot \mathrm{mL}^{-1}$ molybdenum ions; $\left.y=2,4,6,8,10\right)$. **: $p<0.01$ 
进行抗菌和细胞活力实验。同时为了进一步观察钼 离子是否会降低更高浓度铜离子的抗菌性能, 本研 究还探讨了钼离子对浓度为 $100 \mu \mathrm{g} \cdot \mathrm{mL}^{-1}$ 的铜离子 抗菌性能的影响。结果证明: 钼离子确实能够拮抗 高浓度铜离子 $\left(10 \mu \mathrm{g} \cdot \mathrm{mL}^{-1}\right)$ 对人脐静脉内皮细胞的 毒性，同时并不影响铜离子对金黄色葡萄球菌的抗 菌性能。

\section{3 钼掺杂铜硅钙石的抗菌性能和细胞相容性}

为了探究钼掺杂铜硅钙石(10Mo-Cup)的抗菌 性能, 本研究用不同稀释倍数的 10Mo-Cup 细菌培 养基浸提液进行了 S. A. 培养实验。如图 5 所示,

表 1 不同稀释倍数 10Mo-Cup 细菌培养基浸提液离子浓度

Table 1 Ion contents in the extract with 10Mo-Cup bacterial culture medium after different dilutions

\begin{tabular}{lccc}
\hline $\begin{array}{c}\text { Dilutions of } \\
\text { the extract }\end{array}$ & $\begin{array}{c}\mathrm{Cu} \text { ion/ } \\
\left(\mu \mathrm{g} \cdot \mathrm{mL}^{-1}\right)\end{array}$ & $\begin{array}{c}\mathrm{Si} \text { ion/ } \\
\left(\mu \mathrm{g} \cdot \mathrm{mL}^{-1}\right)\end{array}$ & $\begin{array}{c}\text { Mo ion/ } \\
\left(\mu \mathrm{g} \cdot \mathrm{mL}^{-1}\right)\end{array}$ \\
\hline 1 & 709.86 & 88.71 & 243.10 \\
$1 / 40$ & 17.75 & 2.22 & 6.08 \\
$1 / 80$ & 8.87 & 1.11 & 3.04 \\
$1 / 160$ & 4.44 & 0.55 & 1.52 \\
\hline
\end{tabular}


图 $510 \mathrm{Mo}-\mathrm{Cup}$ 细菌培养基浸提液对金黄色葡萄球菌的抑 制作用

Fig. 5 Inhibition effect of extract from 10Mo-Cup bacterial culture medium on Staphylococcus aureus

(a) Number of Staphylococcus aureus after being treated with different dilutions of 10Mo-Cup medium extract; (b) Related antibacterial rate. $*: p<0.05 ; * *: p<0.01$
10Mo-Cup 浸提液的抗菌性能随浸提液的稀释倍数 增大而降低。1/80 的 10Mo-Cup 原始细菌培养基浸 提液(铜离子的浓度为 $8.87 \mu \mathrm{g} \cdot \mathrm{mL}^{-1}$, 表 1)对 S.A 的 抗菌率为 $90.5 \%$ 。因此，当铜离子的释放量高于 $8.87 \mu \mathrm{g} \cdot \mathrm{mL}^{-1}$ 时, $10 \mathrm{Mo}-\mathrm{Cup}$ 细菌浸提液对 S.A.的抑 制率高于 $90 \%$ 。

为了研究 10Mo-Cup 释放的铜离子对细胞的毒 性, 本研究用不同稀释倍数的 $10 \mathrm{Mo}-\mathrm{Cup}$ 细胞培养 液浸提液进行了 HDFs 和 HUVECs 细胞活力实验。 如图 6(a)所示, HDFs 在 1/8 的 10Mo-Cup 原始细

\section{表 2 10Mo-Cup ECM 培养基浸提液 在不同稀释倍数时的离子浓度}

Table 2 Ion contents in the extract with 10Mo-Cup cell culture medium after different dilutions

\begin{tabular}{cccc}
\hline $\begin{array}{c}\text { Dilution of } \\
\text { the extract }\end{array}$ & $\begin{array}{c}\mathrm{Cu} \text { ion/ } \\
\left(\mu \mathrm{g} \cdot \mathrm{mL}^{-1}\right)\end{array}$ & $\begin{array}{c}\mathrm{Si} \text { ion/ } \\
\left(\mu \mathrm{g} \cdot \mathrm{mL}^{-1}\right)\end{array}$ & $\begin{array}{c}\text { Mo ion/ } \\
\left(\mu \mathrm{g} \cdot \mathrm{mL}^{-1}\right)\end{array}$ \\
\hline 1 & 154.35 & 78.85 & 268.57 \\
$1 / 4$ & 38.59 & 19.71 & 67.14 \\
$1 / 8$ & 19.29 & 9.86 & 33.57 \\
$1 / 16$ & 9.65 & 4.93 & 16.79 \\
\hline
\end{tabular}

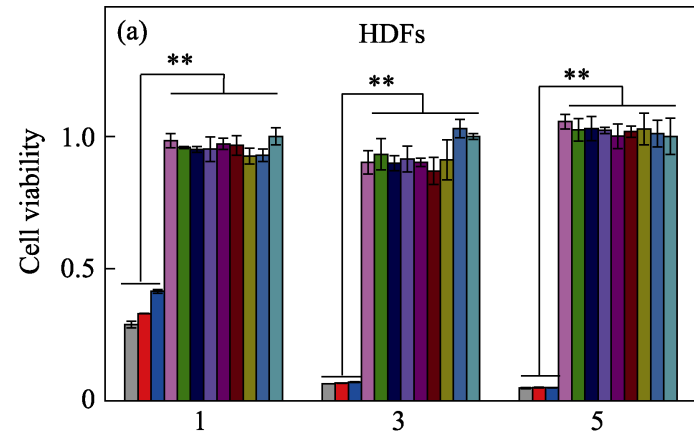

Culture time / d

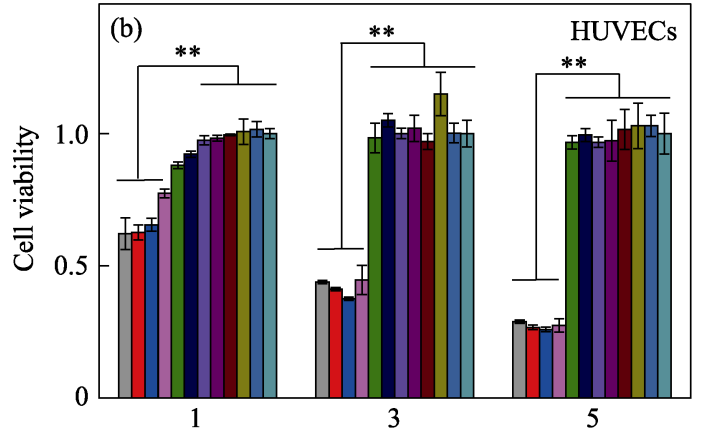

Culture time / d

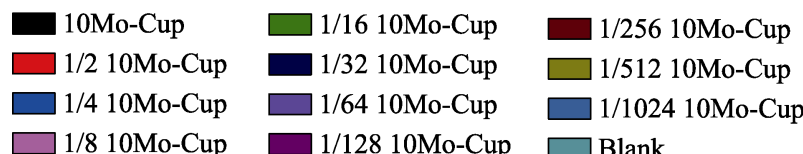

图 6 不同稀释倍数的 $10 \mathrm{Mo}-\mathrm{Cup}$ 细胞培养液浸提液对 (a)HDFs 和(b)HUVECs 活力的影响

Fig. 6 Effects of 10Mo-Cup culture medium extracts with different concentrations on (a) HDFs and (b) HUVECs activity assay. ${ }^{* *}: p<0.01$ 
胞培养液浸提液及更高稀释倍数的浸提液中的存活 率能达到 Blank 组的 $85 \%$ 以上。如图 6(b)所示, $1 / 8$ 的 10Mo-Cup 原始细胞培养液浸提液对 HUVECs 有 明显的毒性, 稀释至 $1 / 16$ 时, 铜离子浓度为 $9.65 \mu \mathrm{g} \cdot \mathrm{mL}^{-1}$ (表 2)及更高稀释倍数时, HUVECs 存 活率能达到 Blank 组的 $90 \%$ 以上。因此, 当铜离子 释放浓度不高于 $9.65 \mu \mathrm{g} \cdot \mathrm{mL}^{-1}$ 时, $10 \mathrm{Mo}-\mathrm{Cup}$ 细胞培 养液浸提液对 HUVECs 有良好的细胞相容性。

含铜生物材料的抗菌性能与铜离子的释放量 呈正相关关系, 对大肠杆菌和金黄色葡萄球菌产生 明显抑制作用时, 最低铜离子浓度分别在 7 和 $11 \mu \mathrm{g} \cdot \mathrm{mL}^{-1[6,17]}$ 。而含铜生物材料的细胞相容性随材 料铜离子的释放量增加而变弱 ${ }^{[18-19]}$, HUVECs 在 铜离子高于 $3.2 \mu \mathrm{g} \cdot \mathrm{mL}^{-1}$ 时存活率低于 $60 \%{ }^{[7]}$ 。因此, 在保证含铜生物材料抗菌性能的同时, 很难避免其 对 HUVECs 的毒性。由于铜钼离子的拮抗作用能降 低高浓度铜离子对细胞的毒性, 使得本研究制备的 10Mo-Cup 在高铜离子浓度 $\left(8.87 \sim 9.65 \mu \mathrm{g} \cdot \mathrm{mL}^{-1}\right.$ ) 释放 下，既能够对金黄色葡萄球菌有 $90 \%$ 以上的抑制效 果, 又能保证 HUVECs 存活率在 $90 \%$ 以上。

\section{3 结论}

通过对铜钿离子组合溶液以及钿掺杂铜硅钙石 (10Mo-Cup)的抗菌性能和细胞相容性的研究得到如 下结论:

1)当铜钼离子组合溶液作用金黄色葡萄球菌和 HUVECs 时, 钼离子能够明显降低高浓度铜离子对 HUVECs 的毒性, 并且不影响铜离子的抗菌性能。

2)采用溶胶凝胶法成功制备了 Mo-Cup, 通过 掺杂方式将钼元素引入到含铜生物材料中。

3) 10Mo-Cup 的铜离子释放浓度在 8.87 $9.65 \mu \mathrm{g} \cdot \mathrm{mL}^{-1}$ 高浓度范围时, 对金黄色葡萄球菌抑 制率达到 90\%以上，且对 HUVECs 没有毒性。

因此, 钼掺杂能够有效降低含铜生物材料的高 浓度铜离子对 HUVECs 的毒性而不影响其抗菌性 能, 为制备兼具优异抗菌性能和良好生物相容性的 含铜生物材料提供了新的路径。

\section{参考文献:}

[1] LI P L, HAN F X, CAO W W, et al. Carbon quantum dots derived from lysine and arginine simultaneously scavenge bacteria and promote tissue repair. Applied Materials Today, 2020, 6(19): 100601.
[2] ZHANG Y, CHANG M L, BAO F, et al. Multifunctional Zn doped hollow mesoporous silica/polycaprolactone electrospun membranes with enhanced hair follicle regeneration and antibacterial activity for wound healing. Nanoscale, 2019, 11(13): 6315-6333.

[3] NING C, WANG X, LI L, et al. Concentration ranges of antibacterial cations for showing the highest antibacterial efficacy but the least cytotoxicity against mammalian cells: implications for a new antibacterial mechanism. Chemical Research in Toxicology, 2015, 28(9): 1815-1822.

[4] TAN S X, TAN S Z, LIU Y L, et al. Preparation and antibacterial property of copper-loaded activated carbon microspheres. Journal of Inorganic Materials, 2010, 25(3): 299-305.

[5] WU C T, ZHOU Y H, XU M C, et al. Copper-containing mesoporous bioactive glass scaffolds with multifunctional properties of angiogenesis capacity, osteostimulation and antibacterial activity. Biomaterials, 2013, 34(2): 422-433.

[6] TIAN T, WU C T, CHANG J. Preparation and in vitro osteogenic, angiogenic and antibacterial properties of cuprorivaite $\left(\mathrm{CaCuSi}_{4} \mathrm{O}_{10}\right.$, Cup) bioceramics. RSC Advances, 2016, 6(51): 45840-45849.

[7] 孔妮. 掺铜硅酸钙生物陶瓷的制备与表征及其促血管化性能的 研究. 上海: 上海交通大学硕士学位论文, 2015.

[8] KONG N, LIN K L, LI H Y, et al. Synergy effects of copper and silicon ions on stimulation of vascularization by copper-doped calcium silicate. Journal of Materials Chemistry B, 2014, 2(8): $1100-1110$.

[9] YANG Z J, LONG T, RAN L W, et al. Molybdenum's biological function and roles in animal production. Journal of Henan University of Science and Technology (Agricultrual Science), 2004(2): 40-43.

[10] WU M J. Molybdenum and human health. Studies of Trace Elements and Health, 2006, 5(23): 66-67.

[11] WANG Q X. Trace element molybdenum and human health. Studies of Trace Elements and Health, 2003, 4(20): 58-59.

[12] LIU M. The effect of molybdenum on human health. China Molybdenum Industry, 2001, 5(25): 43-45.

[13] 田瑶. 四硫代钼酸铵抑制顺铂与人铜伴侣蛋白 Atoxl 的相互作 用. 合肥: 中国科学技术大学硕士学位论文, 2018 .

[14] ALVAREZ H M, XUE Y, ROBINSON C D, et al. Tetrathiomolybdate inhibits copper trafficking proteins through metal cluster formation. Science, 2010, 327(5963): 331-334.

[15] PAN Q, KLEER C G, VAN GOLEN K L, et al. Copper deficiency induced by tetrathiomolybdate suppresses tumor growth and angiogenesis. Cancer Research, 2002, 62(17): 4854-4859.

[16] CHAN N, WILLIS A, KORNHAUSER N, et al. Influencing the tumor microenvironment: a phase II study of copper depletion using tetrathiomolybdate in patients with breast cancer at high risk for recurrence and in preclinical models of lung metastases. Clinical Cancer Research, 2017, 23(3): 666-676.

[17] MIAO Z Z, LI G W, LIU C Z, et al. Study on antibacterial properties of copper-loaded chitosan particles. Journal of Henan Institute of Science and Technology, 2010, 38(2): 78-80.

[18] XU Q, CHANG M L, ZHANG Y, et al. PDA/Cu bioactive hydrogel with "hot ions effect" for inhibition of drug-resistant bacteria and enhancement of infectious skin wound healing. ACS Applied Materials \& Interfaces, 2020, 12(28): 31255-31269.

[19] LI J, ZHAI D, LÜ F, et al. Preparation of copper-containing bioactive glass/eggshell membrane nanocomposites for improving angiogenesis, antibacterial activity and wound healing. Acta Biomater., 2016, 36: 254-266. 